\title{
A Compound Poisson Risk Model with a Two-Step Premium Rule
}

\author{
Mi Jung Song ${ }^{a}$, Jiyeon Lee ${ }^{1, a}$ \\ ${ }^{a}$ Department of Statistics, Yeungnam University
}

\begin{abstract}
We consider a compound Poisson risk model in which the premium rate changes when the surplus exceeds a threshold. The explicit form of the ruin probability for the risk model is obtained by deriving and using the overflow probability of the workload process in the corresponding $M / G / 1$ queueing model.

Keywords: Compound Poisson risk model, ruin probability, two-step premium rule, $M / G / 1$ queue, overflow probability, renewal equation.
\end{abstract}

\section{Introduction}

In this paper, a compound Poisson risk model with a two-step premium rule is considered. We assume that customers' claims arrive according to a Poisson process $\{N(t), t \geq 0\}$ with a parameter $\lambda>0$, and the sizes of the individual claims $\left\{Y_{1}, Y_{2}, \ldots\right\}$ are independent and identically distributed positive random variables with a common distribution function $G(y)=\operatorname{Pr}[Y \leq y]$ and mean $m=\int_{0}^{\infty} y \mathrm{~d} G(y)$. We also assume that $\left\{Y_{1}, Y_{2}, \ldots\right\}$ are independent of $N(t)$. Then the aggregate of all claims up to time $t$, denoted by $S(t)$, can be expressed by

$$
S(t)=\sum_{i=1}^{N(t)} Y_{i}
$$

where $S(t)=0$ if $N(t)=0$.

If the current surplus is below a constant threshold level $b>0$, then the annual premium rate is assumed to be

$$
p_{1}:=\lambda m\left(1+\theta_{1}\right)
$$

where $\theta_{1}>0$ is the relative security loading. When the surplus is above threshold $b$, then dividends are paid at the annual dividend rate $q, 0<q \leq p_{1}$; therefore, we assume that the premium rate is $p_{2}:=p_{1}-q \geq 0$, and then

$$
p_{2}=\lambda m\left(1+\theta_{2}\right)
$$

where $0<\theta_{2}<\theta_{1}$ is the relative security loading.

If $U(t)$ is the surplus in this risk model at time $t$, then we have

$$
U(t)=u+\int_{0}^{t} p(U(s)) \mathrm{d} s-S(t)
$$

\footnotetext{
${ }^{1}$ Corresponding author: Professor, Department of Statistics, Yeungnam University, Kyeongbuk 712-749, Republic of Korea. E-mail: leejy@yu.ac.kr
} 


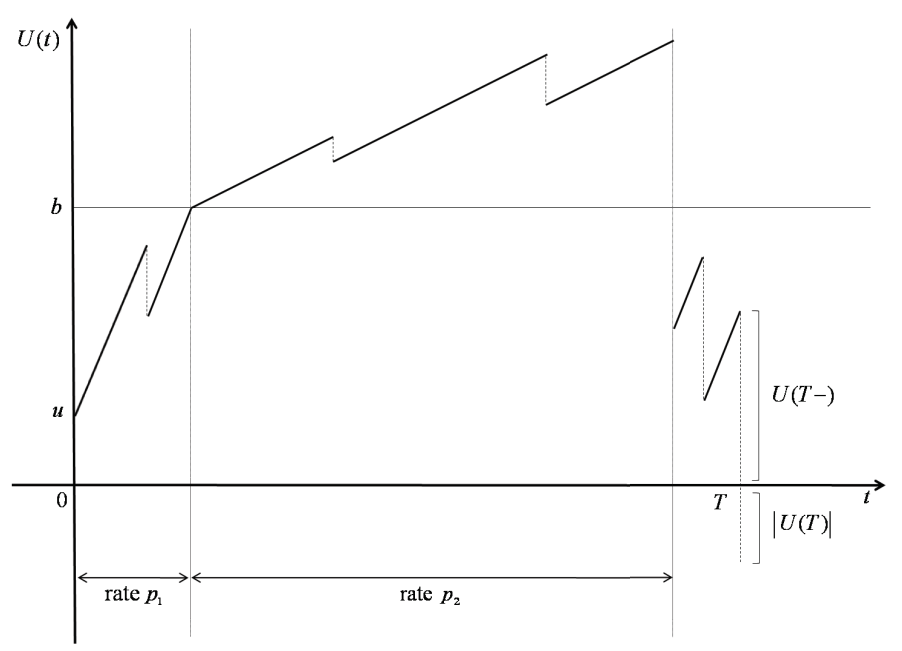

Figure 1: A sample path of the surplus process in the compound Poisson risk model with a two-step premium rule.

where $u=U(0) \geq 0$ is the initial surplus, and

$$
p(x)= \begin{cases}p_{1}, & \text { if } 0 \leq x<b \\ p_{2}, & \text { if } x \geq b\end{cases}
$$

Following previous authors, the (ultimate) ruin time is defined as

$$
T:=\inf \{t>0 \mid U(t)<0\}
$$

where $T=\infty$ if ruin never occurs. A sample path of the surplus process $\{U(t), t \geq 0\}$ is shown in Figure 1.

Lin and Pavlova (2006) analyzed this model by deriving and solving the Gerber-Shiu discounted penalty function introduced by Gerber and Shiu (1998). This useful function allows us to derive results related to joint and marginal distributions of the ruin time $T$, the surplus $U(T-)$ immediately before the ruin time, and the deficit $|U(T)|$ at the ruin time. When $\delta \geq 0$, the Gerber-Shiu discounted penalty function is defined as

$$
m(u)=E\left[e^{-\delta T} v(U(T-),|U(T)|) I(T<\infty) \mid U(0)=u\right],
$$

where $v$ is a nonnegative function of the surplus before ruin and the deficit at ruin, and $I$ is an indicator function. If we let both $\delta=0$ and $v(\cdot, \cdot)=1$, then this function expresses the (ultimate) ruin probability

$$
\psi(u):=\operatorname{Pr}[T<\infty \mid U(0)=u]
$$

which is the main objective of insurance risk models.

Asmussen (2000) pointed out that "Historically, the connection between risk theory and other applied probability areas appears first to have been noted by Prabhu (1961) in a queueing context." Using time reversion, Asmussen and Petersen (1988) made the connection between a risk model with a general premium rate and a dam model with a general release rate; subsequently, they observed that 
the ruin probability can be derived from the stationary distribution of level in the dam; however, the interaction between risk theory and queueing theory has been quite limited.

In this paper, we obtain the ruin probability $\psi(u)$ by applying the overflow probability of the workload process in the $M / G / 1$ queueing model corresponding to a compound Poisson risk model. The duality between the queueing model and the risk model facilitates the derivation of some characteristics of one model from the other. Song and Lee (2011) obtained the probability that the ruin occurs before the surplus reaches a given large value in this risk model. Section 2 reviews several results related to the ordinary $M / G / 1$ workload process and Section 3 describes a modified $M / G / 1$ workload process that furthers our analysis of the surplus process with a two-step premium rule. We also derive the overflow probability that the workload exceeds a certain level for the first time. Section 4 uses the overflow probability of the queueing model to obtain a ruin probability for the risk model, which is consistent with that derived by Lin and Pavlova (2006).

\section{2. $M / G / 1$ Workload Process}

Let $\{V(t), t \geq 0\}$ be the workload process for an ordinary $M / G / 1$ queueing model in which a server works with a service rate $p$ : that is, the workload of the server decreases by $p$ over unit time. Customers arrive according to a Poisson process of rate $\lambda$, and the workloads added by the arriving customers are independent and identically distributed with distribution $G$ and mean $m$. If we rescale time by considering $1 / p$ as the unit of time, then the arrival rate becomes $\lambda / p$, the service rate becomes 1 , and the traffic intensity becomes $\rho:=\lambda \mathrm{m} / \mathrm{p}$. We assume that $\rho<1$.

Let $0 \leq \alpha<\beta<\infty$. For $\alpha \leq x \leq \beta$, and following Lee (2007), we define

$$
T_{x}(\alpha, \beta):=\inf \{t \geq 0 \mid V(t) \notin(\alpha, \beta], V(0)=x\}
$$

to represent the first exit time from $(\alpha, \beta]$ for the process $\{V(t), t \geq 0\}$, starting at $V(0)=x$. We use $G_{e}(x)$ to denote the equilibrium distribution function of $G$, which is

$$
G_{e}(x):=\frac{1}{m} \int_{0}^{x}[1-G(u)] \mathrm{d} u,
$$

and we let

$$
H(x)=\sum_{n=0}^{\infty} \rho^{n} G_{e}^{* n}(x),
$$

where $* n$ is the $n$-fold Stieltjes convolution and $G_{e}^{* 0}(x)$ is the Heaviside function such that $I(x \geq 0)$. Bae et al. (2002) showed that

$$
\operatorname{Pr}\left[V\left(T_{x}(0, \beta)\right)>\beta\right]=1-\frac{H(\beta-x)}{H(\beta)}, \quad 0 \leq x \leq \beta,
$$

meaning that the probability of the process $\{V(t), t \geq 0\}$, starting at $x$, up-crosses $\beta$ before reaching 0 . If we generalize the interval, this expression becomes

$$
\operatorname{Pr}\left[V\left(T_{x}(\alpha, \beta)\right)>\beta\right]=1-\frac{H(\beta-x)}{H(\beta-\alpha)}, \quad \alpha \leq x \leq \beta .
$$


Bae et al. (2001) also obtained the distribution of levels of the process $\{V(t), t \geq 0\}$ at the exit time $T_{x}(0, x)$ for $x>0$, which is given by

$$
\operatorname{Pr}\left[V\left(T_{x}(0, x)\right)>x+l\right]=\frac{\rho\left(H * J_{l}\right)(x)}{H(x)}, \quad l \geq 0,
$$

where

$$
J_{l}(x):=G_{e}(x+l)-G_{e}(l)
$$

and

$$
\left(H * J_{l}\right)(x)=\int_{0-}^{x} J_{l}(x-s) \mathrm{d} H(s) .
$$

Similarly, for any $0 \leq \alpha<x$, we can show that

$$
\operatorname{Pr}\left[V\left(T_{x}(\alpha, x)\right)>x+l\right]=\frac{\rho\left(H * J_{l}\right)(x-\alpha)}{H(x-\alpha)}, \quad l \geq 0 .
$$

Let $\left\{V_{\infty}(t), t \geq 0\right\}$ be the process that is derived from $\{V(t), t \geq 0\}$ when we allow the workload to take negative values. If we use $L_{x}$ to denote the excess amount at the first passage time through $x$ of the process $\left\{V_{\infty}(t), t \geq 0\right\}$, starting at $x$, then we have the following lemma:

Lemma 1. The distribution function of $L_{x}$ is given by

$$
\operatorname{Pr}\left[L_{x} \leq l\right]=1-\rho\left[1-G_{e}(l)\right]
$$

which is independent of $x$.

Proof: From the definition of $L_{x}$, we observe that

$$
L_{x} \equiv V_{\infty}\left(T_{x}(-\infty, x)\right)-x,
$$

where $\equiv$ denotes equality of distribution. It follows from (2.2) that, for any $-\infty<a<x$,

$$
\operatorname{Pr}\left[V_{\infty}\left(T_{x}(a, x)\right)>x+l\right]=\frac{\rho\left(H * J_{l}\right)(x-a)}{H(x-a)} .
$$

Letting $a$ go to $-\infty$ in (2.3) yields

$$
\begin{aligned}
\operatorname{Pr}\left[L_{x}>l\right] & =\lim _{a \rightarrow-\infty} \operatorname{Pr}\left[V_{\infty}\left(T_{x}(a, x)\right)>x+l\right] \\
& =\frac{\rho\left(H * J_{l}\right)(\infty)}{H(\infty)} \\
& =\rho\left[1-G_{e}(l)\right],
\end{aligned}
$$

in which the final equality follows from $H(\infty)=1 /(1-\rho)$ and

$$
\begin{aligned}
\left(H * J_{l}\right)(\infty) & =\int_{0-}^{\infty}\left[1-G_{e}(l)\right] \mathrm{d} H(s) \\
& =\frac{1-G_{e}(l)}{1-\rho}
\end{aligned}
$$

because $H(0-)=0$. 


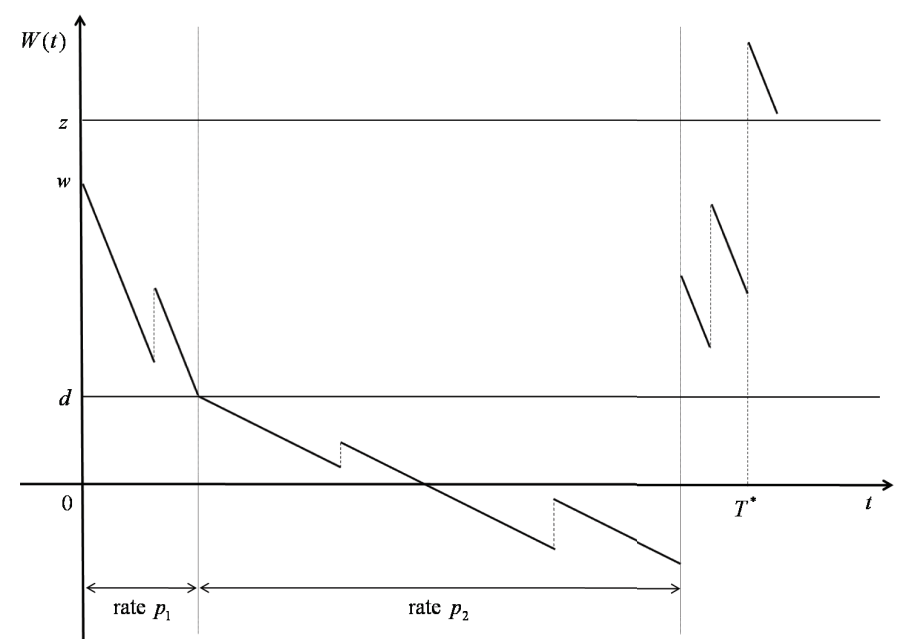

Figure 2: A sample path of the workload process $\{W(t), t \geq 0\}$.

\section{3. $M / G / 1$ Workload Process under a Two-Step Service Rule}

We first specify an $M / G / 1$ queueing model where the workload process is coupled to the surplus process of our risk model. The arrival of customers follows a Poisson process of rate $\lambda$, and each customer brings a job consisting of an amount of work $Y$ that is independent and identically distributed with the distribution function $G$ and mean $m$. A server starts to work on an initial workload of $w$ and decreases that workload at two different service rates: If the current workload exceeds a constant threshold $d$, then the server works at the service rate $p_{1}$. If the workload is less than $d$, the server changes his/her service rate to $p_{2}$. We assume that $\lambda m<p_{1}$ and $\lambda m<p_{2}$. Furthermore, we assume that the workload can take negative values. If the workload belongs to $(d, \infty)$, then the server works with a service rate of $p_{1}$; however, if the workload lies in $(-\infty, d]$, the server works with a service rate of $p_{2}$. Let $\{W(t), t \geq 0\}$ be the workload process of this queueing model. Figure 2 depicts a sample path of the workload process $\{W(t), t \geq 0\}$.

If the workload exceeds some large $z$, then we say that overflow has occurred in this model. Therefore, the overflow time is defined as $T^{*}:=\inf \{t>0 \mid W(t)>z\}$, where $T^{*}=\infty$ if no overflows occur. Thus, for $w \leq z$, the probability that the workload process overflows can be expressed as

$$
\xi(w, z):=\operatorname{Pr}\left[T^{*}<\infty \mid W(0)=w\right] .
$$

Since $\xi(w, z)$ depends on the initial level $w$, we can write

$$
\xi(w, z)= \begin{cases}\xi_{1}(w, z), & \text { if } d<w \leq z \\ \xi_{2}(w, z), & \text { if } w \leq d\end{cases}
$$

\subsection{Overflow probability when $d<w \leq z$}

If the initial workload $w$ lies in $(d, z]$, then the server starts to serve with rate $p_{1}$. Once the workload reaches the lower level $d$, the server changes its service rate to $p_{2}$. Let $\left\{W_{1}(t), t \geq 0\right\}$ be a process obtained from the original process $\{W(t), t \geq 0\}$ by separating the periods during which the service rate is $p_{1}$ and then connecting them together. Therefore, the process $\left\{W_{1}(t), t \geq 0\right\}$ has the state space $(d, \infty)$. Furthermore, let $\left\{W_{2}(t), t \geq 0\right\}$ be formed similarly by separating and connecting the residual 
periods of the process $\{W(t), t \geq 0\}$. Then the process $\left\{W_{2}(t), t \geq 0\right\}$ has the service rate $p_{2}$ and the state space $(-\infty, d]$.

We note that the process $\{W(t), t \geq 0\}$, starting at $w$, can up-cross $z$ either before or after reaching $d$. Since the process $\{W(t), t \geq 0\}$ coincides with $\left\{W_{1}(t), t \geq 0\right\}$ until it down-crosses $d$, the probability that $\{W(t), t \geq 0\}$ up-crosses $z$ before reaching $d$ is equal to the probability that $\left\{W_{1}(t), t \geq 0\right\}$ upcrosses $z$ before reaching $d$. From (2.1), we see that the latter probability is given by

$$
\operatorname{Pr}\left[W_{1}\left(T_{w}(d, z)\right)>z\right]=1-\frac{H_{1}(z-w)}{H_{1}(z-d)},
$$

where $H_{1}(x):=\sum_{n=0}^{\infty} \rho_{1}^{n} G_{e}^{* n}(x)$ with $\rho_{1}:=\lambda m / p_{1}$.

If the process $\{W(t), t \geq 0\}$, starting at $w$, is to up-cross $z$ after reaching $d$, it obviously has to reach $d$ without up-crossing $z$. In this case we decompose $\{W(t), t \geq 0\}$ into two processes $\left\{W_{1}(t), t \geq 0\right\}$ and $\left\{W_{2}(t), t \geq 0\right\}$. The process $\{W(t), t \geq 0\}$ is the same as $\left\{W_{1}(t), t \geq 0\right\}$ until it reaches $d$. Thus, from the Markov property of $\{W(t), t \geq 0\}$, it follows that

$$
\begin{aligned}
& \operatorname{Pr}\left[W\left(T_{w}(d, z)\right)=d, W\left(T_{d}(-\infty, z)\right)>z\right] \\
& =\operatorname{Pr}\left[W_{1}\left(T_{w}(d, z)\right)=d\right] \operatorname{Pr}[\{W(t), t \geq 0\}, \text { starting at } d, \text { up-crosses } z] .
\end{aligned}
$$

Let $L_{x}^{*}$ denote the excess amount at the first passage time through $x$ of the process $\left\{W_{2}(t), t \geq 0\right\}$ which starts at $x \leq d$. By Lemma $1, L_{x}^{*}$ has the distribution function $F(l):=1-\rho_{2}\left[1-G_{e}(l)\right]$ with $\rho_{2}:=\lambda m / p_{2}$. After the process $\{W(t), t \geq 0\}$ reaches $d$, it replicates $\left\{W_{2}(t), t \geq 0\right\}$ until it leaves $(-\infty, d]$. Therefore, if we condition the above expression on $L_{d}^{*}$, we have

$$
\begin{aligned}
\operatorname{Pr} & {[\{W(t), t \geq 0\}, \text { starting at } d, \text { up-crosses } z] } \\
= & \operatorname{Pr}\left[\left\{W_{2}(t), t \geq 0\right\}, \text { starting at } d, \text { up-crosses } z\right] \\
& +\int_{0}^{z-d} \operatorname{Pr}\left[\left\{W_{1}(t), t \geq 0\right\}, \text { starting at } d+l, \text { up-crosses } z\right] \mathrm{d} F(l) \\
= & 1-F(z-d)+\int_{0}^{z-d} \xi_{1}(d+l, z) \mathrm{d} F(l) .
\end{aligned}
$$

Combining (3.1), (3.2) and (3.3), we obtain

$$
\begin{aligned}
\xi_{1}(w, z) & =1-\frac{H_{1}(z-w)}{H_{1}(z-d)}+\frac{H_{1}(z-w)}{H_{1}(z-d)}\left(1-F(z-d)+\int_{0}^{z-d} \xi_{1}(d+l, z) \mathrm{d} F(l)\right) \\
& =1-\frac{H_{1}(z-w)}{H_{1}(z-d)}\left(1-\rho_{2}+\rho_{2} \int_{0}^{z-d}\left[1-\xi_{1}(d+l, z)\right] \mathrm{d} G_{e}(l)\right) .
\end{aligned}
$$

We will now find an explicit expression for the solution of this renewal equation.

Theorem 1. If the initial value of $w$ belongs to $(d, z]$, then the overflow probability of the workload process $\{W(t), t \geq 0\}$ is given by

$$
\xi_{1}(w, z)=1-\frac{\rho_{1}\left(1-\rho_{2}\right) H_{1}(z-w)}{\rho_{2}+\left(\rho_{1}-\rho_{2}\right) H_{1}(z-d)} .
$$

Proof: To simplify our notation, we let

$$
\Phi_{1}(w, z):=1-\xi_{1}(w, z) .
$$


Then (3.4) can be rewritten as

$$
\Phi_{1}(w, z)=\frac{H_{1}(z-w)}{H_{1}(z-d)}\left(1-\rho_{2}+\rho_{2} \int_{0}^{z-d} \Phi_{1}(d+l, z) \mathrm{d} G_{e}(l)\right) .
$$

From this equation we can see that $\Phi_{1}(w, z)$ is a product of $H_{1}(z-w)$ and some function of $z$, which we will call $A(z)$. Therefore we can write

$$
\Phi_{1}(w, z)=H_{1}(z-w) A(z) .
$$

Substituting this equation into (3.5) yields

$$
H_{1}(z-w) A(z)=\frac{H_{1}(z-w)}{H_{1}(z-d)}\left(1-\rho_{2}+\rho_{2} A(z) \int_{0}^{z-d} H_{1}(z-d-l) \mathrm{d} G_{e}(l)\right) .
$$

Solving this equation with respect to $A(z)$ while noting that the relation $\left(G_{e} * H_{1}\right)(x)=\left[H_{1}(x)-1\right] / \rho_{1}$, we have

$$
A(z)=\frac{\rho_{1}\left(1-\rho_{2}\right)}{\rho_{2}+\left(\rho_{1}-\rho_{2}\right) H_{1}(z-d)},
$$

which completes the proof.

We remark that the overflow probability $\xi_{1}(w, z)$ depends on $w$ and $z$ through $z-w$ and $z-d$.

\subsection{Overflow probability when $w \leq d$}

If the initial workload of the process $\{W(t), t \geq 0\}$ is below $d$, then the server starts to serve at rate $p_{2}$. If this process starts at $w \leq d$ and is subsequently to exceed $z$, then $\left\{W_{2}(t), t \geq 0\right\}$ must first exceed $w$, because $\left\{W_{2}(t), t \geq 0\right\}$ replicates $\{W(t), t \geq 0\}$ until they both up-cross $w$. Thus, by conditioning on $L_{w}^{*}$, we have

$$
\begin{aligned}
\xi_{2}(w, z) & =1-F(z-w)+\int_{0}^{d-w} \xi_{2}(w+l, z) \mathrm{d} F(l)+\int_{d-w}^{z-w} \xi_{1}(w+l, z) \mathrm{d} F(l) \\
& =\rho_{2} \int_{0}^{d-w} \xi_{2}(w+l, z) \mathrm{d} G_{e}(l)+\rho_{2}\left(1-G_{e}(z-w)+\int_{d-w}^{z-w} \xi_{1}(w+l, z) \mathrm{d} G_{e}(l)\right) .
\end{aligned}
$$

Theorem 2. If the initial value of $w$ is less than or equal to $d$, then the overflow probability of the workload process $\{W(t), t \geq 0\}$ is given by

$$
\xi_{2}(w, z)=\int_{0}^{d-w} \eta(z-w-l, d-w-l) d H_{2}(l)+\eta(z-w, d-w),
$$

where

$$
\eta(z-w, d-w):=\rho_{2}\left(1-G_{e}(z-w)+\int_{d-w}^{z-w} \xi_{1}(w+l, z) d G_{e}(l)\right) .
$$

Proof: Since $\xi_{1}(w, z)$ is determined by both $z-w$ and $z-d$, it can be expressed in terms of $z-w$ and $d-w$. Thus we can write the right-side of (3.7) as a function of $z-w$ and $d-w$. Substitute (3.7) 
into (3.6). We notice that the overflow probability $\xi_{2}(w, z)$ is also determined by $z-w$ and $d-w$, so it can be expressed as $\xi_{2}(w, z):=B(z-w, d-w)$ for some function $B(\cdot, \cdot)$. Let $y=d-w$ and $h(y):=B(z-d+y, y)$. Then we can rewrite (3.6) as

$$
h(y)=\rho_{2} \int_{0}^{y} h(y-l) \mathrm{d} G_{e}(l)+\eta(z-d+y, y),
$$

which is a type of renewal equation. Asmussen $(2003$, p.146) showed that the unique solution to this renewal equation is given by

$$
h(y)=\int_{0}^{y} \eta(z-d+y-l, y-l) \mathrm{d} H_{2}(l)+\eta(z-d+y, y),
$$

where $H_{2}(x)=\sum_{n=0}^{\infty} \rho_{2}^{n} G_{e}^{* n}(x)$ with $\rho_{2}=\lambda m / p_{2}$. Thus it follows that

$$
\xi_{2}(w, z)=\int_{0}^{d-w} \eta(z-w-l, d-w-l) \mathrm{d} H_{2}(l)+\eta(z-w, d-w) .
$$

\section{Ruin Probabilities}

We will now use overflow probability $\xi(w, z)$ to obtain the ruin probability $\psi(u)$ of the compound Poisson risk model with a two-step premium rule. If we let $u:=z-w$ and $b:=z-d$, then for $t \geq 0$, we have

$$
U(t) \equiv z-W(t)
$$

and then $\psi(u)=\xi(w, z)$. Thus it follows that

$$
\begin{aligned}
\psi_{1}(u) & :=\left.\psi(u)\right|_{0 \leq u<b} \\
& =\xi_{1}(w, z) \\
& =1-\frac{\theta_{2} H_{1}(u)}{\left(1+\theta_{1}\right)-\left(\theta_{1}-\theta_{2}\right) H_{1}(b)}
\end{aligned}
$$

and

$$
\begin{aligned}
\psi_{2}(u) & :=\left.\psi(u)\right|_{u \geq b} \\
& =\xi_{2}(w, z) \\
& =\int_{0}^{u-b} \zeta(u-l) \mathrm{d} H_{2}(l)+\zeta(u),
\end{aligned}
$$

where

$$
\zeta(u):=\frac{1}{1+\theta_{2}}\left[\int_{u-b}^{u} \psi_{1}(u-l) \mathrm{d} G_{e}(l)+1-G_{e}(u)\right],
$$

because $p_{i}=\lambda m\left(1+\theta_{i}\right)$. These results are consistent with the ruin probability obtained by Lin and Pavlova (2006). 
Example 1. We assume that the size of each claim is exponentially distributed with mean $m$. Then for $i=1,2$ we have

$$
H_{i}(x)=\frac{1+\theta_{i}-e^{-\gamma_{i} x}}{\theta_{i}},
$$

where $\gamma_{i}:=\theta_{i} /\left\{m\left(1+\theta_{i}\right)\right\}$. Thus it follows that

$$
\psi_{1}(u)=1-\frac{\theta_{2}\left(1+\theta_{1}-e^{-\gamma_{1} u}\right)}{\left(1+\theta_{1}\right) \theta_{2}+\left(\theta_{1}-\theta_{2}\right) e^{-\gamma_{1} b}}, \quad 0 \leq u<b
$$

Since $G_{e}(x)=1-\exp (-x / m)$, we obtain

$$
\zeta(u)=\frac{\theta_{1} e^{-\left[\gamma_{1} b+\frac{1}{m}(u-b)\right]}}{\left(1+\theta_{1}\right) \theta_{2}+\left(\theta_{1}-\theta_{2}\right) e^{-\gamma_{1} b}}
$$

and then

$$
\psi_{2}(u)=\frac{\theta_{1} e^{-\left[\gamma_{1} b+\gamma_{2}(u-b)\right]}}{\left(1+\theta_{1}\right) \theta_{2}+\left(\theta_{1}-\theta_{2}\right) e^{-\gamma_{1} b}}, \quad u \geq b .
$$

\section{References}

Asmussen, S. (2000). Ruin Probabilities, World Scientific, Singapore.

Asmussen, S. (2003). Applied Probability and Queues, 2nd ed., Springer, New York.

Asmussen, S. and Petersen, S. S. (1988). Ruin probabilities expressed in terms of storage processes, Advances in Applied Probability, 20, 913-916.

Bae, J., Kim, S. and Lee, E. Y. (2001). The virtual waiting time of the $M / G / 1$ queue with impatient customers, Queueing Systems, 38, 485-494.

Bae, J., Kim, S. and Lee, E. Y. (2002). A $P_{\lambda}^{M}$-policy for an $M / G / 1$ queueing system, Applied Mathematical Modeling, 26, 929-939.

Gerber, H. U. and Shiu, E. S. W. (1998). On the time value of ruin, North American Actuarial Journal, 2, 48-78.

Lee, J. (2007). First exit times for compound Poisson dams with a general release rule, Mathematical Methods for Operations Research, 65, 169-178.

Lin, X. S. and Pavlova, K. P. (2006). The compound Poisson risk model with a threshold dividend strategy, Insurance: Mathematics and Economics, 38, 57-80.

Prabhu, N. U. (1961). On the ruin problem of collective risk theory, Annals of Mathematical Statistics, 32, 757-764.

Song, M. J. and Lee, J. (2011). Ruin probability in a compound Poisson risk model with a two-step premium rule, Communications on the Korean Statistical Society, 18, 433-443. 